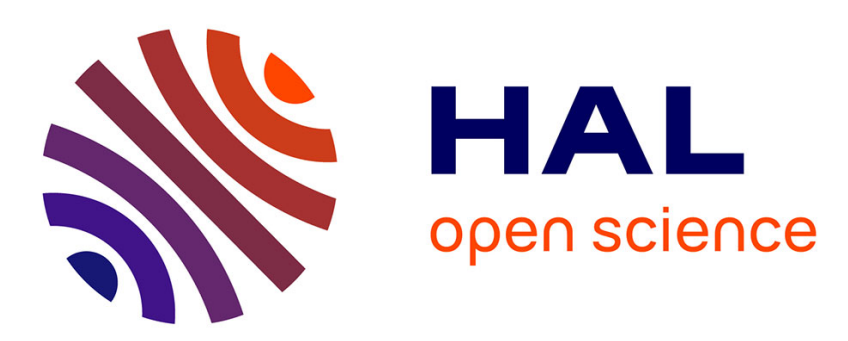

\title{
RRAM-based non-volatile SRAM cell architectures for ultra-low-power applications
}

\author{
Hussein Bazzi, Adnan Harb, Hassen Aziza, Mathieu Moreau, Abdallah
}

Kassem

\section{> To cite this version:}

Hussein Bazzi, Adnan Harb, Hassen Aziza, Mathieu Moreau, Abdallah Kassem. RRAM-based nonvolatile SRAM cell architectures for ultra-low-power applications. Analog Integrated Circuits and Signal Processing, 2021, 106 (2), pp.351-361. 10.1007/s10470-020-01587-z . hal-03504286

\section{HAL Id: hal-03504286 \\ https://hal.science/hal-03504286}

Submitted on 29 Dec 2021

HAL is a multi-disciplinary open access archive for the deposit and dissemination of scientific research documents, whether they are published or not. The documents may come from teaching and research institutions in France or abroad, or from public or private research centers.
L'archive ouverte pluridisciplinaire HAL, est destinée au dépôt et à la diffusion de documents scientifiques de niveau recherche, publiés ou non, émanant des établissements d'enseignement et de recherche français ou étrangers, des laboratoires publics ou privés. 


\title{
RRAM-based Non-Volatile SRAM Cell Architectures for Ultra-Low-Power Applications
}

\author{
Hussein Bazzi ${ }^{1,2}$, Adnan Harb ${ }^{3}$, Senior Member, IEEE, Hassen Aziza ${ }^{2}$, Mathieu Moreau ${ }^{2}$, Abdallah Kassem ${ }^{4}$ \\ ${ }^{1}$ Department of Electrical and Electronics Engineering, Lebanese International University, Al Khiara, Bekaa, Lebanon \\ ${ }^{2}$ IM2NP-UMR CNRS 7334, Aix-Marseille University, Marseille, France \\ ${ }^{3}$ Department of Electrical and Electronics Engineering, International University of Beirut, Beirut, Lebanon \\ ${ }^{4}$ Department of Electrical and Computer and Communication Eng., Notre Dame University-Louaize, Zouk Mosbeh, Lebanon \\ Corresponding author: adnan.harb@b-iu.edu.lb
}

\begin{abstract}
Static Random-Access Memories (SRAMs) have flourished in the memory market relying on their speed, power consumption and compatibility with standard CMOS process technology. Conventional SRAMs are characterized by volatility, limiting their role in applications where non-volatility is essential. Non-Volatile SRAMs (NVSRAMs) represent an appealing solution, where Resistive RAM (RRAM) can act as a non-volatile element for SRAM. RRAM relies on the basic physical phenomenon of operation called resistive switching. This paper presents different NVSRAM structures, while exploring their principle of operation. Also, a comparison in terms of area, speed, power consumption and design complexity is presented for three NVSRAM memory cells implemented in a 130-nm high voltage CMOS technology from STMicroelectronics.
\end{abstract}

Index Terms - Computing, Resistive Switching Memory (RRAM), Non-Volatile Static Random-Access Memory (NVSRAM), Area, Power Consumption, Switching Speed.

\section{INTRODUCTION}

In the recent years, several unconventional memory technologies have been explored [1]. This trend to investigate new technologies is driven by the growing demand for low power, high speed, and low-cost Non-Volatile Memory (NVM) technology suitable for today's market [2]. In this context, Resistive Random-Access Memory (RRAM) is a promising candidate among the next-generation NVM technologies [3]. This technology is characterized by its simple structure, fast switching speed, high scalability, and the capability to be integrated at the Back-End-Of-Line (BEOL) of a conventional CMOS process [4].

Nowadays, memory subsystem directly affects the performance of computing system. The hierarchy of the memory subsystem can be classified under numerous categories: Static Random-Access Memory (SRAM), Dynamic Random-Access Memory (DRAM), and flash memories. All these memories are charge-based. In SRAM, the data is stored as charges at the nodes of the cross-coupled inverters [5-9].

In general, charge-based memories suffer from performance degradation with the down scaling reaching deep nanoscale (10-nm node and beyond). This problem affects the reliability, endurance, and the noise margin of SRAM memory. In this context, emerging memory technologies and specifically RRAM, have been under extensive research and development for charge-based memory replacement [10].

In the current mobile market, large number of electronic systems and applications depend on semiconductor solid state memories. The performance needed by new electronic devices pushes towards ultra-low power storage media. To fulfill this demand and answer tomorrow's challenges, RRAM emerging devices are considered to be part of these appraised memory technologies [11].

SRAM can benefit from this development, as it can be combined with RRAM technology to create a new hybrid structure called Non-Volatile SRAM (NVSRAM) [12]. NVSRAM array is designed to be electrically stable at the low supply voltages. Moreover, in order to achieve small chip size, the memory cell size must be as small as possible [13].

In this paper, 3 NVSRAM memory cells (6T2R, 10T1R and $8 \mathrm{~T} 1 \mathrm{R})$ are presented and compared. These structures are implemented in a $130-\mathrm{nm}$ high voltage CMOS technology from STMicroelectronics [14]. In section II, the oxide-based RRAM technology is presented. In section III, the traditional 6T SRAM is presented and proposed NVSRAM memory cell architectures are detailed. In Section IV, NVSRAM cell simulation results are presented. Also, the layout of all NVSRAM structures is provided along with benchmarking results. In section $\mathrm{V}$, projections are provided to estimate the chip area of each NVSRAM cell at a memory array level. Section VI presents concluding remarks.

\section{OXIDE-BASED RRAM TECHNOLOGY}

Oxide-based RRAM technology (OxRAM) is based on a Metal-Insulator-Metal (MIM) structure, where the metal electrodes sandwich the insulator layer made of an oxide material as shown in Fig. 1. RRAM devices switching mechanism rely on the formation/dissolution of a conductive filamentary in the insulator layer. This filamentary is based on oxygen vacancies migration between the metal electrodes [15]. Filamentary switching based on Transition Metal Oxides (TMOs) has attracted a lot of attention [16]. In the pristine state, OxRAM device is initially in a very high resistive state and require an electrical soft-breakdown (Forming) equivalent to the injection of oxygen vacancies in the insulating layer. Forming is executed once in the life of an OxRAM cell, where a high voltage is applied across the cell to switch from HighResistance Sate (HRS) to a Low-Resistance State (LRS) [17]. 


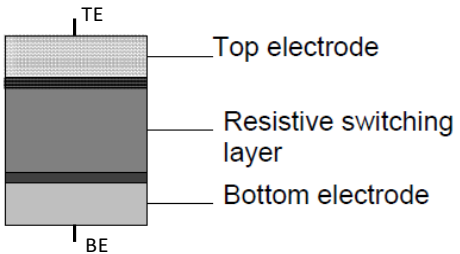

(a)

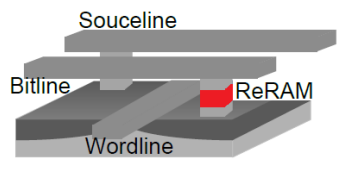

(b)
Fig. 1. Basic RRAM structure.

RRAM devices demonstrate reliable resistive switching between HRS and a LRS. The switching from HRS to LRS is termed "SET," and the switching from LRS to HRS is termed "RESET." An electrical stimulus (i.e. voltage pulse) applied across the OxRAM device terminals is needed to trigger the switching mechanism. Note that the considered OxRAM technology is compatible with the conventional CMOS fabrication environment and process temperatures [18].

From a circuit simulation point of view, in this work, the Stanford model [19] is used to model the OxRAM element. Stanford model is a physics-based compact model, taking into consideration the temperature effect, the timing effect and variability. These effects are observed in many actual OxRAM cells [20]. Temperature dependency is an important feature of the Stanford model. Indeed, the conductive filament rupture, or dissolution occurs under the effect of a significant change in temperature based on the fundamental concept of Joule heating [21]. During the RESET process and by increasing the applied voltage, the temperature steadily rises until a value called the critical temperature. Above this critical temperature, the conductive filament dissolution/rupture takes place at a fast rate inducing a High Resistance State (HRS) of the device. During the SET process, the temperature rises due to the increase in the $\mathrm{CF}$ radius. In the Stanford model [19], the applied voltage, as shown in Fig. 2, directly affects the temperature change in the $\mathrm{CF}$ radius. A temperature peak is observed at each SET and RESET sequence.

The correspondence between the experimental data and the simulation data proves that the model captures very well the randomness of the resistance levels during the SET and RESET processes of different RRAM cells. Fig. 3 depicts the simulated I-V curves of Stanford model for 10 SET cycles compared to the experimental data. The variation is mainly due to the random generation of oxygen vacancy.

The hysteresis loop of an OxRAM represented by its current-voltage (I-V) characteristic is shown in Fig. 4. The I$\mathrm{V}$ characteristic shows a sharp SET/RESET transition. OxRAM device usually comes with a select transistor establishing the 1T1R structure. The current limitation is imposed by the select transistor during SET operation in order to limit the current flowing through the device. The current limit is called the "compliance current". Based on the linear curve presented in Fig. 2, the $\mathrm{V}_{\mathrm{SET}}$ value needed to switch from HRS to LRS state is equal to $1.6 \mathrm{~V}$, while the $V_{\text {RESET }}$ value required to switch back to HRS state is equal to $-1 \mathrm{~V}$.

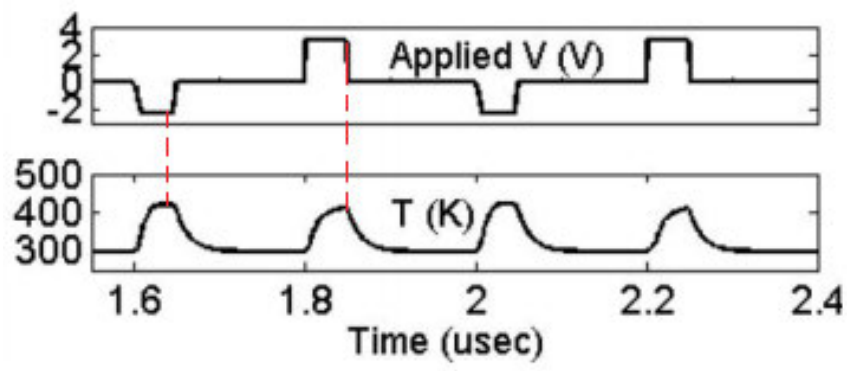

Fig. 2. Stanford model simulated temperature evolution as a function of the applied voltage [22]

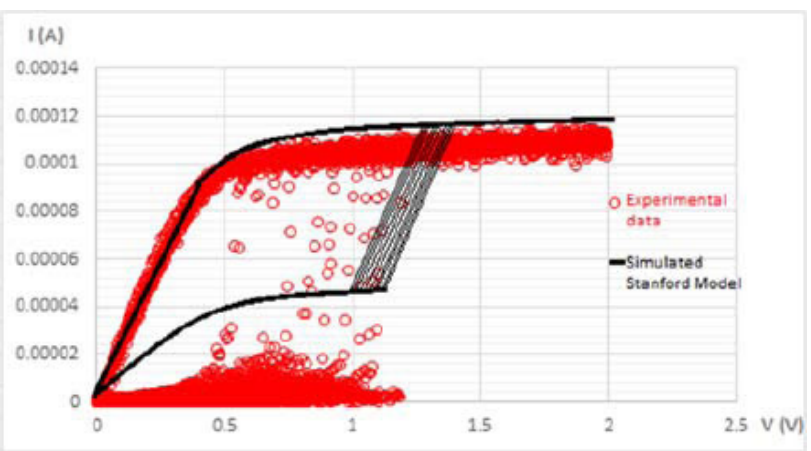

Fig. 3: Single-cell Stanford model variability for 10 SET cycles [21]

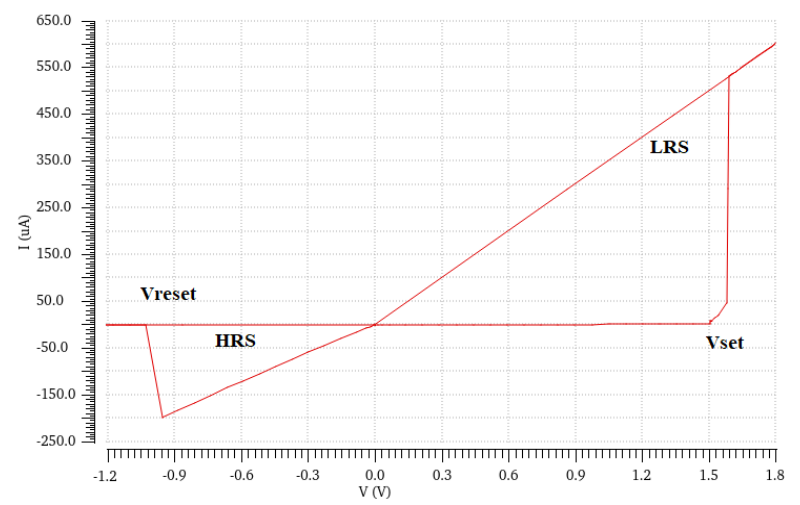

Fig. 4. OxRAM I-V characteristic

\section{NVSRAM STRUCTURES}

In RRAM-based NVSRAMs, the SRAM cell is combined with a RRAM device. Data nodes of the SRAM cell are connected to the resistive elements allowing data backup operations while the SRAM normal mode operation is preserved (i.e. HOLD, WRITE and READ operations). A STORE operation allows to save the SRAM data in the RRAM device and a RESTORE operation updates SRAM data. STORE and RESTORE operations are performed before power off and after power on respectively [14]. STORE/RESTORE operations in NVSRAMs are the counterparts of WRITE/READ operations in SRAMs.

\section{A. Conventional 6T SRAM memory cell}

The conventional SRAM structure follows the crosscoupled inverters structure presented in Fig.5. The 3 main operations in a SRAM are HOLD, READ and WRITE. In an 


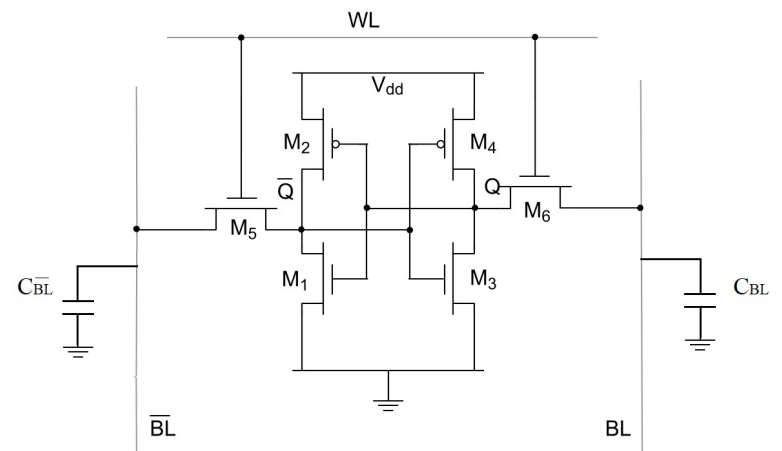

Fig. 5. 6T SRAM cell

array of SRAM memory cells, rows are called Word Lines (WLs) and columns are called Bit Lines (BLs). WLs are connected to the gates of the SRAM select transistors (M5 and M4). WRITE are READ operations in the memory cells are accomplished through the bit lines [23]. During a HOLD operation, the WL is deactivated to disconnect $\mathrm{BL}$ and $\mathrm{BL} /$ from the SRAM cell. As a result, data is held in the latch structure on nodes $\mathrm{Q}$ and $\mathrm{Q} /$. When WL is activated, READ and WRITE operations can be executed.

Three NVSRAM cells are presented in the next sections. These NVSRAM cells consist of 6 transistors and 2 RRAM devices (6T2R), 10 transistors and 1 RRAM device (10T1R), and 8 transistors and 1 RRAM device (8T1R).

\section{B. 6T2R memory cell}

For optimal performances, the addition of RRAM devices should be well located to avoid any malfunction in the storing and restoring operations [24]. To this end, RRAM devices take PMOS transistor locations in the 6T2R memory cell as presented in Fig. 6. This solution reduces the number of transistors of the memory cell compared to other NVSRAM cells with Transmission Gates (TGs). PMOS transistors are added on each side of the SRAM cell to form TGs. The main benefit of this topology is related to the use of TGs. A transmission gate is mainly used to enhance the switching speed, especially in storing " 1 " as the single NMOS in the traditional 6T SRAM is not able to pass strong " 1 ". The $(W / L)$ ratio of NMOS and PMOS is kept as the standard minimum with $\mathrm{W}=150 \mathrm{~nm}$ and $\mathrm{L}=130 \mathrm{~nm}$ to limit the current during RRAM programming cycles [25].

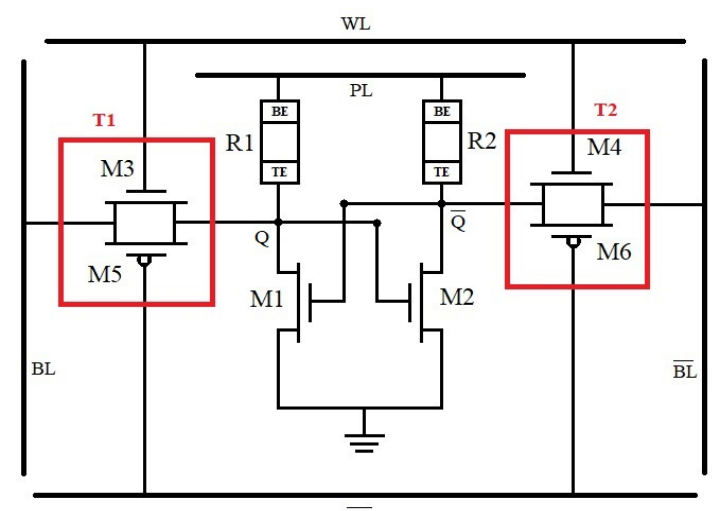

$\overline{\mathrm{WL}}$
In the HOLD state, no write or read operation is permitted as the WL is grounded. During a STORE operation, TGs T1 and $\mathrm{T} 2$ are $\mathrm{ON}$, connecting the bit lines to the RRAM top electrodes (TE). In the process of storing " 1 " on R1, BL is set to $\mathrm{V}_{\mathrm{DD}}$ and $\mathrm{BL} /$ and $\mathrm{PL}$ are grounded. In this configuration, M1 transistor is OFF and a current flows through T1 to R1. The voltage applied on TE decreases the resistance of R1, thus writing a logical "1" (R1 is SET). Note that when storing "1" in R1 on the first cycle, " 0 " is stored in R2 in a second cycle. In the process of storing " 0 " on $\mathrm{R} 1$, the $\mathrm{BL}$ is set to " 0 " and $\mathrm{BL} /$ and $\mathrm{PL}$ are set to $\mathrm{V}_{\mathrm{DD}}$. In this configuration, $\mathrm{M} 1$ transistor is $\mathrm{ON}$ and a current flows through $\mathrm{R} 1$ to $\mathrm{T} 1$. The voltage applied on BE increases R1 resistance, thus writing " 0 " on R1 (R1 is RESET). Note that due to the design topology used, STORE and WRITE operations are exhibited simultaneously.

During a RESTORE operation, WL is set to $0 \mathrm{~V}$. PL line is set to $\mathrm{V}_{\mathrm{DD}}$, inducing a current through the RRAM devices. Data stored in RRAM devices are restored (i.e. nodes $\mathrm{Q}$ and $\mathrm{Q} /$ are updated). If R1 is in LRS state and R2 in HRS state, the storage node Q remains at " 1 " and node Q/ is discharged through M2. If R1 is in HRS state and R2 in LRS state, Q discharges back to " 0 " and Q/ is charged to " 1 ".

\section{10T1R memory cell}

The 10T1R structure presented in Fig. 7 follows the basic SRAM cross-coupled structure and only one RRAM device is used. T1 and T2 are used as access gates similar to the 6T2R. RRAM top electrode is connected to the output of the control transmission gate T3. As usual, STORE is executed before powering down the cell. NMOS and PMOS transmission gates sizing follows the standard minimal design rule with $\mathrm{W}$ $=150 \mathrm{~nm}$ and $\mathrm{L}=130 \mathrm{~nm}$ [26-27].

HOLD operation is similar to the 6T2R cell where TGs T1, T2, and T3 are OFF. During the WRITE operation, T1 and $\mathrm{T} 2$ are $\mathrm{ON}$ and $\mathrm{T} 3$ is OFF to complete the programming of the nodes (Q and Q/). During STORE, R1 is accessed by turning ON T3 allowing Q voltage to reach TE. For instance, in the process of storing " 1 ", PL and WL are grounded. As a result, $\mathrm{R} 1$ cell is SET since the node Q was already programed to " 1 ".

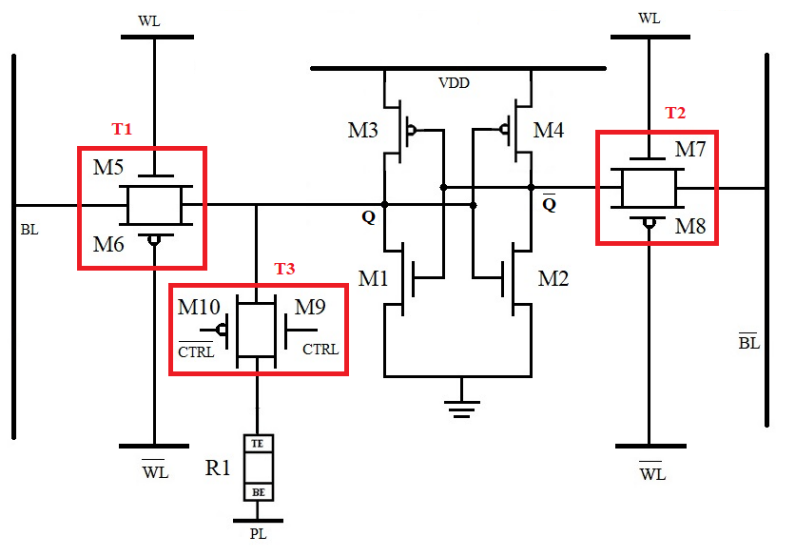

Fig. 7. 10T1R NVSRAM Cell

Fig. 6.6T2R NVSRAM Cell 
During a RESTORE operation, the reading circuitry is connected to the bit lines. PL is set to $\mathrm{VDD}_{\mathrm{DD}} / 2$ and $\mathrm{TG} T 3$ is $\mathrm{ON}$. The bit stored in $\mathrm{R} 1$ is restored, and nodes ( $\mathrm{Q}$ and $\mathrm{Q} /$ ) are updated. RESTORE state in this design rely on one RRAM device. In this structure, only one SET/RESET operation is needed, since there is one RRAM device, where in the previous structure 2 operations were needed.

\section{Novel 8T1R NVSRAM cell}

The proposed $8 \mathrm{~T} 1 \mathrm{R}$ structure presented in Fig. 8 is characterized by a minimal number of control signals (i.e. BL and WL). M3 and M4 represent the traditional SRAM access transistors. STORE/RESTORE operation is executed through two additional transistors M5 and M6. The OxRAM device is connected to M5 transistor (TE) and to the node Q/ (BE).

During Forming, BL and WL/ are set high, WL and BL/ are set low. In this stage, the OxRAM can be formed in one step (M6 is ON: direct connection between BL and the OxRAM). STORE operation is preceded by a WRITE operation. During WRITE " 0 ” operation, BL and WL/ are set low, WL and BL/ are set high. RESET is the next stage after WRITE " 0 ", where WL and BL are set low. In the Pre-charge stage (normal operation), BL and $\mathrm{BL} /$ are set to $\mathrm{V}_{\mathrm{DD}} / 2(0.9 \mathrm{~V})$. Regarding the READ operation, it is executed through WL which is set high.

After STORE (SET/RESET) and READ operations, all the signals are turned OFF including $\mathrm{V}_{\mathrm{DD}}$. STORE operation is required to retain the data after the power is down.

\section{Simulation Results}

6T2R, 10T1R and 8T1R NVSRAM simulation results are proposed in this section, followed by comparison of the different NVSRAM architectures. Voltage pulses (WL, BL and PL) are set to $1.8 \mathrm{~V}$ which is compatible with the available voltage supply $\mathrm{V}_{\mathrm{DD}}$ for the considered technology.

\section{A. 6T2R NVSRAM}

Fig. 9 presents the 6T2R NVSRAM cell operation waveforms. Timings are voluntarily relaxed for memory cell operation understanding. First, the OxRAM cell R2 is RESET and R1 cell is SET.

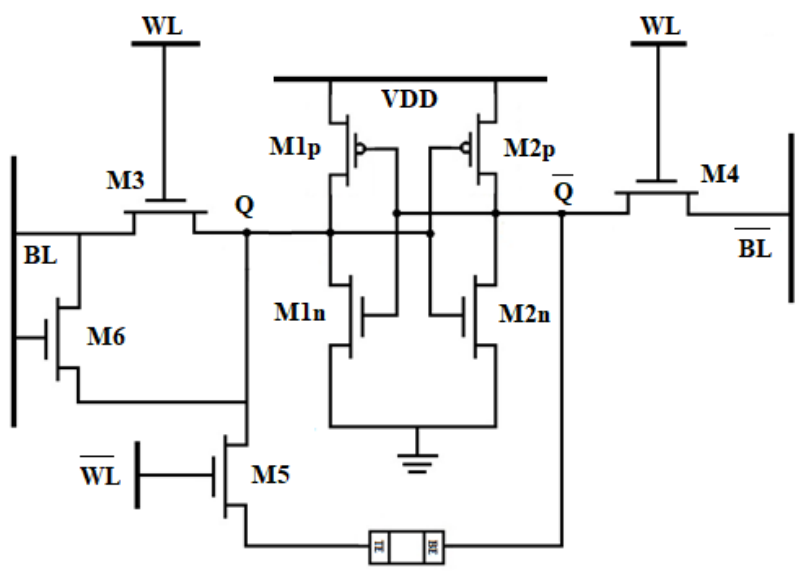

Fig. 8. 8T1R NVSRAM Cell

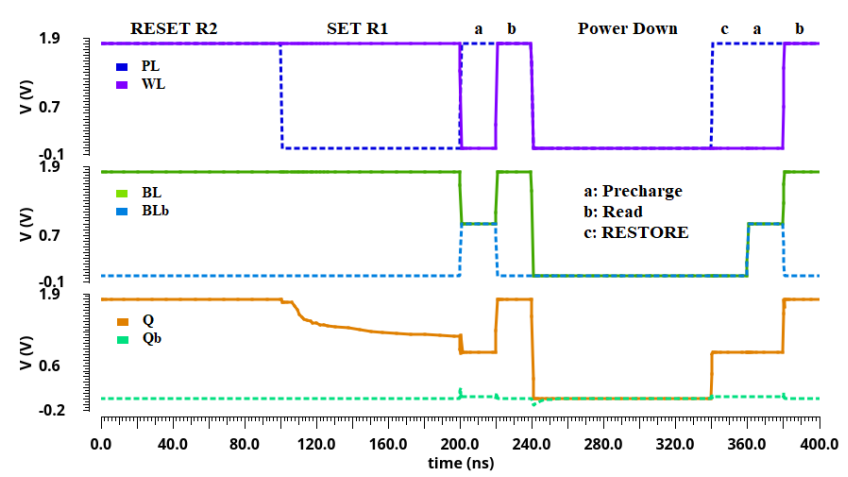

Fig. 9. 6T2R NVSRAM timing waveforms.

During OxRAM programming operations, WL is set High and PL is kept at $\mathrm{V}_{\mathrm{DD}}$ during RESET and connected to ground during SET. During SET, Q voltage decreases to $\sim 0.9 \mathrm{~V}$, while Q/ stays at $0 \mathrm{~V}$. After SET and RESET, a pre-charge and a read operation are executed. During the pre-charge stage, $\mathrm{BL}$ and $\mathrm{BL} /$ are set to $\mathrm{VDD} / 2$ and the $\mathrm{WL}$ is grounded. Result of the read operation is seen through the charge/discharge of BL capacitances. Q and Q/ kept their previous values equal to 1.8 and $0 \mathrm{~V}$ respectively.

At $240 \mathrm{~ns}$ the memory cell is powered down (i.e. all voltages are set to $0 \mathrm{~V}$ ). RESTORE operation starts at $340 \mathrm{~ns}$ $\left(\mathrm{PL}=\mathrm{V}_{\mathrm{DD}}\right.$ and $\left.\mathrm{WL}=0\right)$ to recover $\mathrm{Q}$ and $\mathrm{Q} /$ values from the OxRAM devices. Following the RESTORE operation, a precharge and READ are performed from 360 ns to check $Q$ and $\mathrm{Q} /$ values. $\mathrm{Q}$ and $\mathrm{Q} /$ values equal 1.8 and $0 \mathrm{~V}$ respectively confirming the proper operation of the RESTORE cycle. Fig. 10 shows the 6T2R cell layout with an area occupancy equals to $32.6 \mu \mathrm{m}^{2}$. Static noise margin of the SRAM cell depends on the Cell Ratio (CR), supply voltage and also pull up ratio. Cell ratio is the ratio between sizes of the driver transistor to the load transistor during the read operation. Pull up ratio is also nothing but a ratio between sizes of the load transistor to the access transistor during write operation. Driver transistor is responsible for $70 \%$ value of the SNM. SNM, which affects both read margin and write margin, is related to the threshold voltages of the NMOS and PMOS devices in SRAM cells [28].

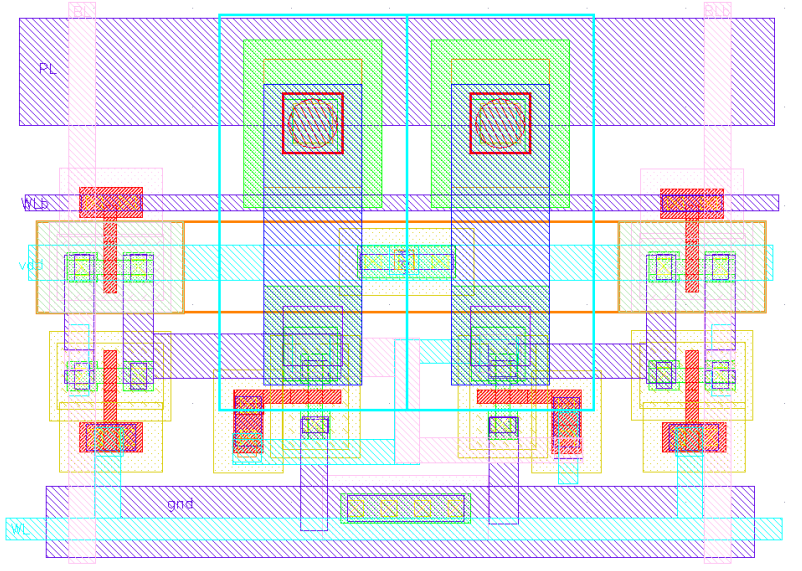

Fig. 10. 6T2R NVSRAM layout 
Fig. 11 presents the Static Noise Margin (SNM) of the 6T2R cell for different SET/RESET duration (in the range [50ns-1 $1 \mu \mathrm{s}])$. The SNM is obtained using the butterfly method where $\mathrm{Q}$ voltage is measured against $\mathrm{Q} /$ voltage and vice versa [29]. The SNM increases with the increase of the STORE Pulse (SP) to reach $365 \mathrm{mV}$. Table I shows the impact of SP parameter on the 6T2R parameters (SET current, Ron, SET energy, switching speed and SNM). The energy consumed to store " 1 " as this value increases from 1.97 to $77.11 \mathrm{pJ}$.

\section{B. IOT1R NVSRAM}

Fig. 12 presents the timing waveform of the 10T1R NVSRAM cell. The 10T1R timing waveform starts with a WRITE operation then a STORE is performed before power down, which is mandatory to RESTORE data. RESTORE, pre-charge and read timing are kept to 20 ns. Fig. 12 shows that $\mathrm{Q}$ and $\mathrm{Q} /$ values are equal to 1.8 and $0 \mathrm{~V}$ respectively indicating that RESTORE is working properly. Fig. 13 shows the 10T1R cell layout, where the area occupied is $40 \mu \mathrm{m}^{2}$.

\section{8T1R NVSRAM}

Fig. 14 presents the 8T1R NVSRAM cell operation waveforms. During Forming, BL, WL/ and $\mathrm{V}_{\mathrm{DD}}$ are set high to form the OxRAM device, where the voltage applied to BL is $5 \mathrm{~V}$. BL is first pre-charged to $\mathrm{V}_{\mathrm{DD}}$ while $\mathrm{BL} /$ is grounded and the access transistors (M5) is activated by applying a pulse on WL/. In a second step, power signal is reconnected (with a value of $\mathrm{V}_{\mathrm{DD}} / 2$ ) and $\mathrm{WL} /$ signal is disabled. During the pre-charge (after RESTORE), BL and BL/ are set to $\mathrm{V}_{\mathrm{DD}} / 2(0.9 \mathrm{~V})$. READ after RESTORE follows the conventional signal sequence, where WL is set high and WL/ is set low.

The sequence of operation coming after power OFF consists in pre-charging the storage node $\mathrm{Q}$ according to the state of the OxRAM device, with BL set to $0.5 \mathrm{~V}$. For instance, if the OxRAM device is in HRS, the voltage at the node $Q$ is lower than the $V_{\text {th }}$ of $M_{1 n} / M_{2 n}$.

Accordingly, when the power signal is reactivated to $\mathrm{V}_{\mathrm{DD}} / 2$, the current coming from the supply, through the PMOS $\left(\mathrm{M}_{2 \mathrm{p}}\right)$ device of the inverter, charges the node $\mathrm{Q} /$ to $\mathrm{V}_{\mathrm{DD}} / 2$. Conversely, if the OxRAM device is in LRS, the voltage of the node $\mathrm{Q}$ is higher than the $\mathrm{V}_{\text {th }}$ of $\mathrm{M}_{1 \mathrm{n}}$ and the current coming from the power signal $\left(\mathrm{V}_{\mathrm{DD}} / 2\right)$ charges the node $\mathrm{Q}$ to a higher voltage than the $\mathrm{V}_{\text {th }}$ of $\mathrm{M}_{1 n}$. Fig. 15 shows the $8 \mathrm{~T} 1 \mathrm{R}$ cell layout, where the area occupied is $45 \mu \mathrm{m}^{2}$. As already mentioned, the main advantage of this structure is the use of a minimal number of signals (BL, WL and $\mathrm{V}_{\mathrm{DD}}$ ). Also, this NVSRAM cell offers low power consumption, low chip area, and the Forming operation is executed in one step.

However, the drawback of this structure is related to the RESTORE operation complexity, which is executed in two steps. During the first step, $\mathrm{V}_{\mathrm{DD}}$ is adjusted to $\mathrm{V}_{\mathrm{DD}} / 2$ to RESTORE the right values at the nodes $\mathrm{Q}$ and $\mathrm{Q} /$, thus $\mathrm{BL}$ and $\mathrm{BL} /$ cannot interfere with nodes $\mathrm{Q}$ and $\mathrm{Q} /$. WL and WL/ are both grounded in the second step of the RESTORE operation. $\mathrm{BL}$ and $\mathrm{BL} /$ are adjusted to $0.5 \mathrm{~V}$ (below $\mathrm{V}_{\text {th }}$ of the
NMOS). When the power signal is reactivated $\left(\mathrm{V}_{\mathrm{DD}} / 2\right)$, the current coming from the supply through the PMOS device $\mathrm{M}_{1 \mathrm{p}}$ of the inverter tries to charge the node $\mathrm{Q}$ to $\mathrm{V}_{\mathrm{DD}} / 2$. Yet, since the $\mathrm{M}_{\mathrm{n} 1}$ is $\mathrm{ON}$, the charges previously built up on the node $\mathrm{Q}$ by $\mathrm{PMOS} \mathrm{M}_{1 \mathrm{p}}$ discharge to ground.

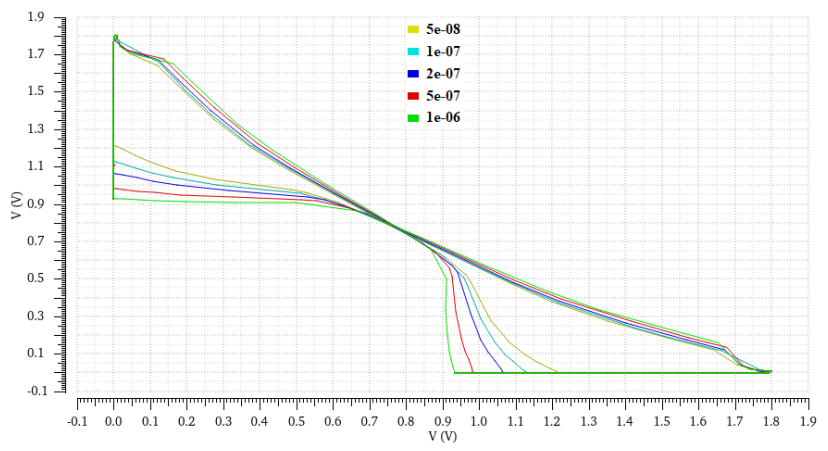

Fig. 11. 6T2R SNM for different STORE Pulses varying in the range [50ns-1us].

TABLE I: IMPACT OF the STORE Pulse ON THE 6T2R CELL PARAMETERS

\begin{tabular}{|c|c|c|c|c|c|}
\hline SP (ns) & $\begin{array}{c}\text { SET current } \\
(\mu \mathrm{A})\end{array}$ & $\begin{array}{c}\text { RoN } \\
(\mathrm{K} \Omega)\end{array}$ & $\begin{array}{c}\text { off/on } \\
\text { Ratio }\end{array}$ & $\begin{array}{c}\text { SET energy } \\
(\text { Store “1”, pJ) }\end{array}$ & $\begin{array}{c}\text { SNM } \\
(\mathrm{mV})\end{array}$ \\
\hline 50 & 30.525 & 59 & 6.36 & 1.97 & 226 \\
\hline 100 & 33.3 & 54 & 6.95 & 4.85 & 262 \\
\hline 200 & 36.396 & 49.4 & 7.59 & 11.79 & 293 \\
\hline 500 & 41.63 & 43.2 & 8.68 & 33.72 & 336 \\
\hline 1000 & 47.678 & 37.7 & 9.95 & 77.11 & 365 \\
\hline
\end{tabular}

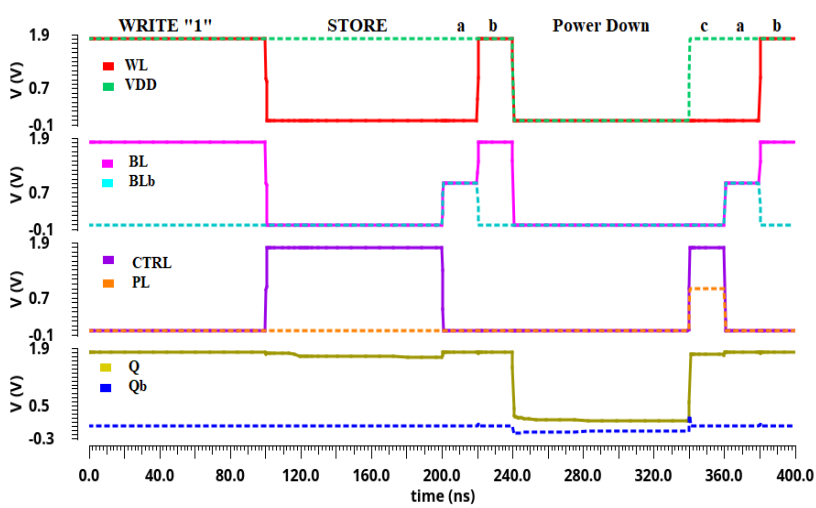

Fig. 12. 10T1R NVSRAM timing waveforms

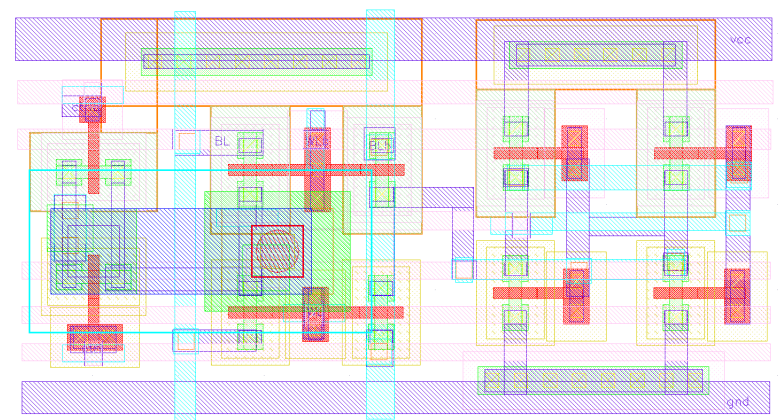

Fig. 13. 10T1R NVSRAM layout 

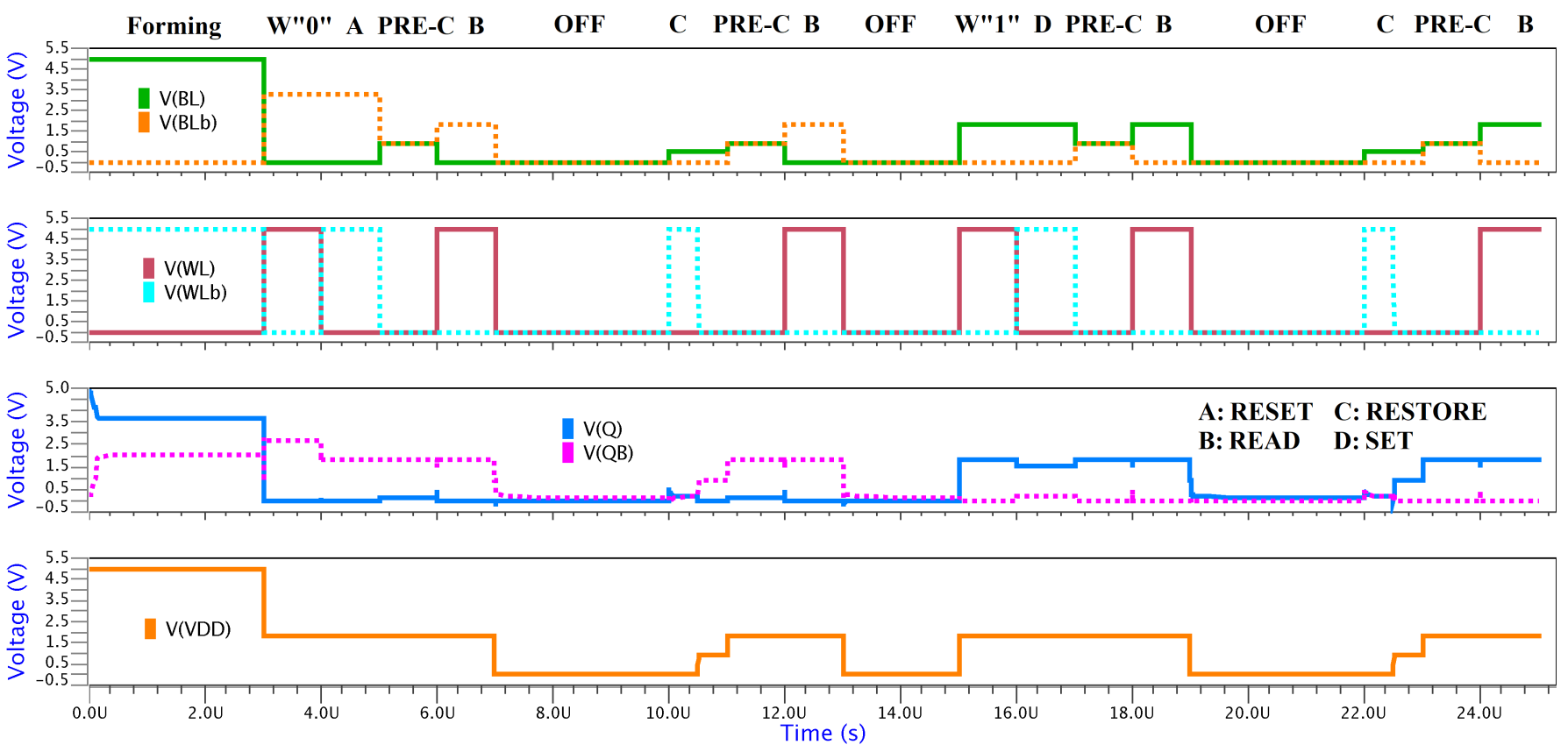

Fig. 14. 8T1R NVSRAM Timing Waveforms

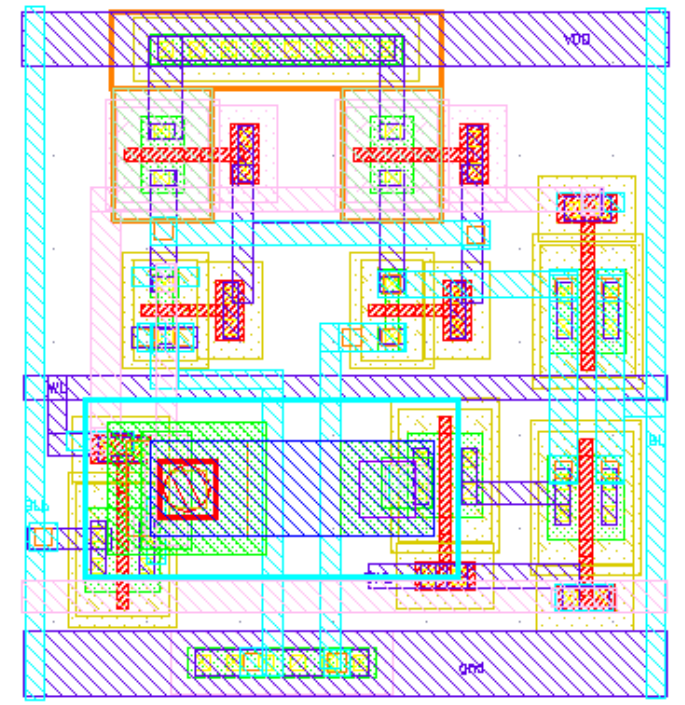

Fig. 15. 8T1R NVSRAM layout

\section{NVSRAMs Comparison}

A comparison between the $6 \mathrm{~T} 2 \mathrm{R}, 10 \mathrm{~T} 1 \mathrm{R}$ and $8 \mathrm{~T} 1 \mathrm{R}$ cells is essential to assess the performance of the different topologies. Table II shows a comparison between the three NVSRAM structures. The comparison is based on four essential parameters including the LRS resistance (RoN), the SNM, the SET energy and the area. The 10T1R cell has higher LRS than the 6T2R and 8T1R. The SET energy of the $6 \mathrm{~T} 2 \mathrm{R}$ and $10 \mathrm{~T} 1 \mathrm{R}$ is very close, but the 10T1R STORE operation is executed just before shutdown. Same as the 8T1R, SET energy is significantly higher, since the final reached LRS is low. The area occupied by the 10T1R is larger $(+22.7 \%)$ since there is a higher number of transistors. $8 \mathrm{~T} 1 \mathrm{R}$ area is $38 \%$ larger than $6 \mathrm{~T} 2 \mathrm{R}$, since transistors used have higher $\mathrm{W} / \mathrm{L}$ ratio. These results indicate that the $6 \mathrm{~T} 2 \mathrm{R}$ cell has an advantage in terms of area, but the 10T1R and 8T1R cells avoid additional power consumption because WRITE and STORE operations are separated.

\section{NVSRAM CELL ARRAY}

In a memory array configuration, NVSRAM cells are arranged to share connections in horizontal rows and vertical columns. The horizontal lines are the Word Lines (WLs), while the vertical lines along which data flow into and out of cells are called Bit Lines (BLs). A cell is accessed for reading or writing by selecting its row and column [30]. Each NVSRAM cell has the capability of storing "0" or "1".

An NVSRAM array is similar to a SRAM array. It consists of multiple cells, where each cell is designed following a specified structure as shown in Fig. 16. When a NVSRAM array is powered-up, initially each individual memory cell acquires either a logic " 0 " or logic " 1 " value according to data stored in the RRAM cells. Also, maximizing the noise margin of NVSRAM cells is a critical factor as it is directly related to the symmetry of the memory cell [31].

TABLE II: COMPARISON BETWEEN NVSRAM CELLS

\begin{tabular}{|c|c|c|c|c|c|c|}
\hline Structure & $\begin{array}{c}\text { RoN } \\
(\mathrm{K} \Omega)\end{array}$ & $\begin{array}{c}\text { SET energy } \\
(\mathrm{pJ})\end{array}$ & $\begin{array}{c}\text { SNM } \\
(\mathrm{mV})\end{array}$ & $\begin{array}{c}\text { Area } \\
\left(\mu \mathrm{m}^{2}\right)\end{array}$ & $\begin{array}{c}\text { Restore } \\
\text { method }\end{array}$ & $\begin{array}{c}\text { Non- } \\
\text { volatility }\end{array}$ \\
\hline 6T2R [25] & 54 & 4.85 & 365 & 32.6 & $\begin{array}{c}\text { Differ- } \\
\text { ential }\end{array}$ & Real-time \\
\hline 10T1R [26] & 65 & 4.14 & 400 & 40 & $\begin{array}{c}\text { Single- } \\
\text { ended }\end{array}$ & $\begin{array}{c}\text { Before } \\
\text { shutdown }\end{array}$ \\
\hline 8T1R & 5.15 & 52.6 & 420 & 45 & $\begin{array}{c}\text { Single- } \\
\text { ended }\end{array}$ & $\begin{array}{c}\text { Before } \\
\text { shutdown }\end{array}$ \\
\hline
\end{tabular}


The three NVSRAM cell architectures are used to build a 4x4 (16-bit) elementary SRAM array presented in Fig. 14. First, the functionality of each memory array is validated. Then, the area of the memory arrays is evaluated. Table III shows a comparison between the three different NVSRAM arrays. The area of the 6T2R, 10T1R and 8T1R NVSRAM arrays is approximately $572 \mu \mathrm{m}^{2}, 720 \mu \mathrm{m}^{2}$ and $806 \mu \mathrm{m}^{2}$ respectively. $6 \mathrm{~T} 2 \mathrm{R}$ array has better density $\left(35.75 \mu \mathrm{m}^{2} / \mathrm{bit}\right)$ than the $10 \mathrm{~T} 1 \mathrm{R}$ array $\left(45 \mathrm{~mm}^{2} / \mathrm{bit}\right)$ and the $8 \mathrm{~T} 1 \mathrm{R}$ array (50.375 $\mu^{2} /$ bit) [32]. To be fully functional, the memory array has to be associated with the surrounding circuitry.

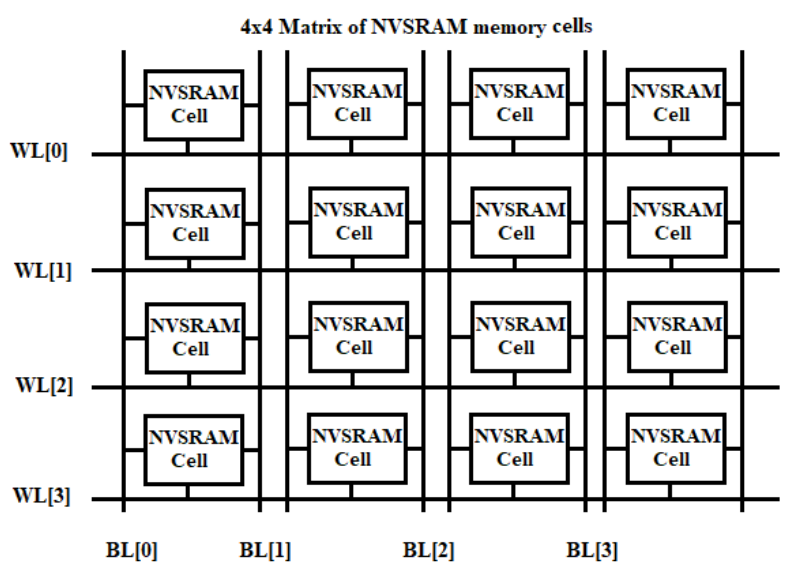

Fig. 16. NVSRAM cell array

TABLE III: COMPARISON BETWEEN NVSRAM ARRAYS

\begin{tabular}{|c|c|c|c|c|}
\hline $\begin{array}{c}\text { Arrays } \\
(16-\mathrm{bit})\end{array}$ & $\begin{array}{c}\text { Width } \\
(\mu \mathrm{m})\end{array}$ & $\begin{array}{c}\text { Height } \\
(\mu \mathrm{m})\end{array}$ & $\begin{array}{c}\text { Area } \\
\left(\mu \mathrm{m}^{2}\right)\end{array}$ & $\begin{array}{c}\text { Bit Density } \\
\left(\mu \mathrm{m}^{2} / \mathrm{bit}\right)\end{array}$ \\
\hline 6T2R & 26.8 & 21.2 & 568 & 35.5 \\
\hline 10T1R & 34.8 & 18.8 & 655 & 41 \\
\hline 8T1R & 27.4 & 29.3 & 803 & 50.18 \\
\hline
\end{tabular}

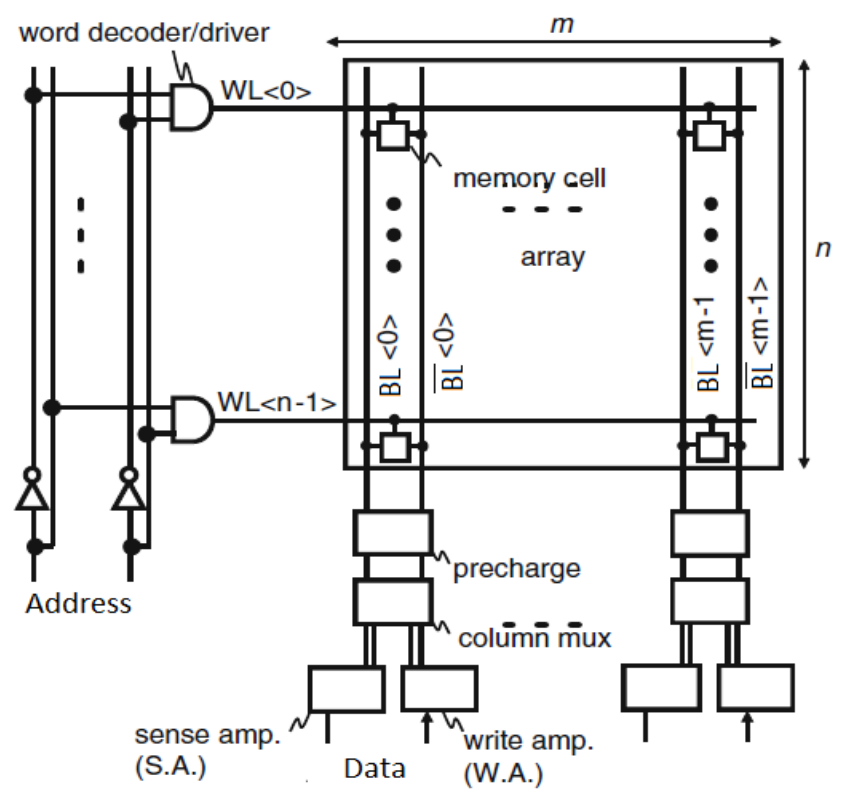

Fig. 17. Basic full NVSRAM cell array structure [28]
Fig. 17 shows a basic full NVSRAM cell array structure including $\mathrm{n}$ rows and $\mathrm{m}$ columns. A word decoder selects one WL based on addresses configuration. Column decoder control circuits consist in numerous peripheral circuits including pre-charge circuits, column multiplexers, sense amplifiers, write amplifiers, etc. READ operation is initiated by selecting the word line. Before this step, the pre-charge circuit increases the bit-line voltage to $\mathrm{V}_{\mathrm{DD}}$ or $\mathrm{V}_{\mathrm{DD}} / 2$. Circuits delivering the logic levels are located at the edge of the bit lines (write amplifiers). After transferring WRITE data to the bit lines, WRITE operation is finalized by turning off the word line [33-34].

\section{CONCLUSION}

This paper compares three NVSRAM cells, including 6T2R, 10T1R and 8T1R cells. All structures are based on the traditional 6T SRAM and implemented in 130-nm technology. TGs are added to 6T2R and 10T1R structures to enhance the overall performance. 6T2R structure achieves low area compared to 10T1R and 8T1R cells. STORE and WRITE operations can be executed separately in 10T1R and 8T1R cells. 8T1R cell introduces a novel structure with a minimal number of control signals. Three NVSRAM array based on the proposed NVSRAM cells are presented with area estimation projections.

\section{ACKNOWLEDGMENT}

I would like to acknowledge the support of the Lebanese International University and Aix-Marseille University, along with the support of Campus France (Eiffel scholarship).

\section{REFERENCES}

[1] H.-T. Lue, Y.-H. Hsiao, K.-Y. Hsieh, S.-Y. Wang, T. Yang, K.-C. Chen, and C.-Y. Lu, "Scaling feasibility study of planar thin floating gate (FG) NAND Flash devices and size effect challenges beyond 20 nm," in IEDM Tech. Dig., 2011, pp. 203-206.

[2] D. Ielmini, "Resistive Switching Memories Based on Metal Oxides: Mechanisms, Reliability and Scaling." Semiconductor Science and Technology, vol. 31, no. 6, 2016, p. 063002.

[3] J. Akerman, "Applied physics: Toward a universal memory," Science, vol. 308, no. 5721, pp. 508-510, Apr. 22, 2005.

[4] R. Waser and M. Aono, "Nanoionics-based resistive switching memories," Nat. Mater., vol. 6, no. 11, pp. 833-840, Nov. 2007.

[5] A. Banerjee, "Ultra-Low-Power Embedded SRAM Design for BatteryOperated and Energy-Harvested IoT Applications." Green Electronics, 2018.

[6] L. Sterpone, et al., "A new reconfigurable clock-gating technique for low power SRAM-based FPGAs," 2011 Design, Automation \& Test in Europe, Grenoble, 2011, pp. 1-6.

[7] P. Singh, et al., "Ultra-Low Power, Process-Tolerant 10T (PT10T) SRAM with Improved Read/Write Ability for Internet of Things (IoT) Applications.” J. Low Power Electron. Appl. 2017, 7, 24.

[8] C. Dou, et al., 2017 IEEE 12th International Conference on ASIC (ASICON).

[9] J.-M. Portal, et al., "RRAM-based FPGA for "An Overview of NonVolatile Flip-Flops Based on Emerging Memory Technologies," Journal of Electronic Science and Technology, vol. 12, no. 2, 2014, pp. 173-181.

[10] H. S. P. Wong, and S. Salahuddin, "Memory leads the way to better computing," Nat. Nanotechnol., vol. 10, pp. 191-194, Mar. 2015.

[11] Meena, J.S., Sze, S.M., Chand, U. et al. Nanoscale Res Lett (2014) 9: 526. 
[12] H. Bazzi, et al., "Novel RRAM CMOS Non-Volatile Memory Cells in 130nm Technology," 2018 International Conference on Computer and Applications (ICCA), Beirut, 2018, pp. 390-393.

[13] A. Benoist, et al., "28nmadvancedCMOS resistive RAM solution as embedded non-volatile memory." In 2014 IEEE International Reliability Physics Symposium, pages 2E.6.1-2E.6.5, June 2014.

[14] H. Bazzi, et al., "Novel RRAM CMOS Non-Volatile Memory Cells in 130nm Technology," ICCA, Beirut, 2018, pp. 390-393.

[15] R. Waser, "Resistive non-volatile memory devices (Invited Paper)," Microelectron. Eng., vol. 86, pp. 1925-1928, 2009.

[16] S. Clima, et, al, IEEE Electron Dev. Lett, Vol.36, Iss.8, pp.769-771, 2015.

[17] Y. Chen and C. Petti, "ReRAM technology evolution for storage class memory application," 2016 46th European Solid-State Device Research Conference (ESSDERC), Lausanne, 2016, pp. 432-435.

[18] Zhu L, et al., "An overview of materials issues in resistive randomaccess memory", Journal of Materiomics (2015).

[19] Jinfeng Kang, et al., "Peking University-Stanford University Resistive Random-Access Memory (RRAM) SPICE Model Version: 2.0Beta", Patent Pending., Copyright Peking University and Stanford University, 2015 All rights reserved, April 13th, 2015.

[20] B. Hajri, el al., "Oxide-based RRAM models for circuit designers: A comparative analysis," 2017 12th International Conference on Design \& Technology of Integrated Systems in Nanoscale Era (DTIS), Palma de Mallorca, 2017, pp. 1-6.

[21] B. Hajri, et al., "RRAM Device Models: A Comparative Analysis with Experimental Validation," in IEEE Access, vol. 7, pp. 168963-168980, 2019.

[22] X. Guan, et al., "A SPICE compact model of metal oxide resistive switching memory with variations," IEEE Electron Device Lett., vol. 33, no. 10, pp. 1405-1407, Oct. 2012.

[23] A. Mazreah, et al., "A Low Power SRAM Base on Novel Word-Line Decoding", World Academy of Science, 2008.

[24] H. Bazzi, et al., "Design of Hybrid CMOS Non-Volatile SRAM Cells in 130nm RRAM Technology," 2018 30th International Conference on Microelectronics (ICM), Sousse, Tunisia, 2018, pp. 228-231.

[25] S. Majumdar, et al., "Programming scheme-based optimization of hybrid 4T-2R OxRAM NVSRAM", Semiconductor Science and Technology, vol. 32, pp. 094008, 2017.

[26] W. Wei, et al., "Design of a Nonvolatile 7T1R SRAM Cell for Instanton Operation." IEEE Transactions on Nanotechnology, vol. 13, no. 5, 2014, pp. 905-916.

[27] J. Mounica, et al., "Design of a nonvolatile 8T1R SRAM Cell for instant-on operation." International Journal of Electrical and Computer Engineering (IJECE), Vol. 6, No. 3, June 2016, pp. $1183 \sim 1189$

[28] S. Birla, et al., "Static Noise Margin Analysis of Various SRAM Topologies". International Journal of Engineering and Technology, vol 3, no. 3, 2011, pp. 304-309.

[29] A. Pavlov, el al., "CMOS SRAM Circuit Design and Parametric Test in Nano-Scaled Technologies". Intel Corporation, University of Waterloo, 2008 Springer Science and Business Media B.V., pp:1 202.

[30] A. Gurjar, et al., "An Analytical Approach to Design VLSI Implementation of Low Power, High Speed SRAM Cell Using Submicron Technology." International Journal of VLSI design \& Communication Systems (VLSICS), 2011. 2(4): p. 143-153.

[31] B. S. Le, et al., "SRAM Cell for High Noise Margin and Soft Errors Tolerance in Nanoscale Technology." 2014 International Conference on Computing, Management and Telecommunications (ComManTel), 2014, doi:10.1109/commantel.2014.6825586.

[32] U. Guin, et al., "Detecting Recycled SoCs by Exploiting Aging Induced Biases in Memory Cells." IEEE International Symposium on Hardware Oriented Security and Trust, May 2019.

[33] Ishibashi, Koichiro, and Kenichi Osada. Low Power and Reliable SRAM Memory Cell and Array Design. Springer, 2011.

[34] C. Shin, et al., "Advanced MOSFET Designs and Implications for SRAM Scaling", Electrical Engineering and Computer Sciences University of California at Berkeley, pp. 1-3, May 2012, Technical Report Number: UCB/EECS-2012-50. 